\title{
Chronoamperometric Versus Galvanostatic Preparation of Manganese Oxides for Electrochemical Capacitors
}

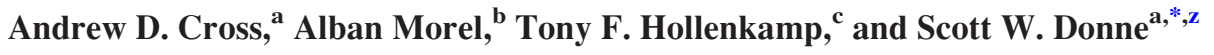 \\ ${ }^{a}$ Discipline of Chemistry, University of Newcastle, Callaghan, NSW 2308, Australia \\ ${ }^{b}$ Ecole Polytechnique de l'université de Nantes, 44306 Nantes Cedex 3, France \\ ${ }^{c}$ CSIRO Energy Technology, PO Box 312, Clayton South, VIC 3169, Australia
}

\begin{abstract}
Chronoamperometric and galvanostatic methods of manganese dioxide electrodeposition have been compared in this work for their ability to produce high specific capacitance manganese dioxide electrodes for supercapacitor applications. When directly compared in terms of the charge passed (and hence the mass of manganese dioxide deposited) and the timeframe of electrodeposition, chronoamperometry most often led to superior performing electrodes. In the best case, an electrode was prepared that attained $2986 \mathrm{~F} / \mathrm{g}$. The results of this work were interpreted in terms of the manganese dioxide deposition mechanism and its impact on deposit morphology. Furthermore, it was concluded that the loss of charge during electrodeposition, as either the result of $\mathrm{Mn}^{3+}$ intermediate species diffusing away from the electrode, or the competing oxygen evolution process, significantly influenced the ability to estimate the amount of manganese dioxide prepared.
\end{abstract}

(C) 2011 The Electrochemical Society. [DOI: 10.1149/1.3625581] All rights reserved.

Manuscript submitted April 18, 2011; revised manuscript received June 28, 2011. Published August 16, 2011.

\section{Introduction}

The importance of energy and energy storage-- One of the major concerns faced by the modern world is the delivery of a constant and efficient supply of energy. The combustion of fossil fuels such as petroleum, natural gas and coal has for many years been the traditional source of energy, and indeed accounted for $85 \%$ of the world's primary sources of energy. ${ }^{1}$ Fossil fuels are not without their drawbacks; indeed, in recent years, concern over the emission of greenhouse gases produced from combustion of fossil fuels, and their suspected role in climate change, as well as the limited supply of oil, coal and natural gas reserves has led to an increased focus on developing more renewable sources of energy. These renewable sources (solar, wind, geothermal, etc.) tend to be implemented on the smaller scale, and deliver power intermittently. To counteract this, any excess energy produced must be stored, so as to be utilised during power sags and compensate for the decreased output of the primary power generator.

Energy storage devices.-With current levels of technology, there are many methods of energy storage, including mechanical (hydroelectric, flywheel and compressed air storage) and thermal (thermal reservoirs such as water, and molten salts of fluorides, chlorides and nitrates) methods. These are relatively low-grade, with efficiencies in the order of $50-90 \%$. $^{2,3}$

Storage as chemical energy is also an alternative; however, combustion of hydrocarbons is also somewhat inefficient, with the typical efficiency of an internal combustion engine in the range of $35 \%$. Electrochemical storage and conversion in devices such as batteries, fuel cells and capacitors, however, is highly efficient and reversible in many cases. The relative merits of each can be summarised using a typical Ragone plot. $^{4}$ Batteries and fuel cells have a relatively high specific energy, but a low specific power. Conversely, capacitors have a low specific energy and a high specific power, making them well suited for applications requiring high current in a short time period. Also included in this category are supercapacitors, or electrochemical capacitors, which have similar power densities as conventional capacitors, but energy densities several orders of magnitude larger. The focus of this work is on further improving the energy density of electrochemical capacitors.

Materials for electrochemical capacitors.-Existing commercial electrochemical capacitors are composed of activated carbon electrodes with an organic electrolyte. They exhibit high reversibility in charge/discharge cycling due to the mechanism of charge storage in the electric double layer. Despite the fact that organic electrolytes

\footnotetext{
* Electrochemical Society Active Member.

z E-mail: scott.donne@newcastle.edu.au
}

are capable of achieving relatively high cell voltages $(2.0-4.0 \mathrm{~V})$, one of their major disadvantages, along with their flammability, toxicity and relatively high cost, is their high resistivity. This is due to larger electrolyte ions, which causes a decreased ionic mobility, which in turn results in a somewhat lower specific power. As a result, electrochemical capacitor systems utilising aqueous electrolytes have received considerable attention, despite their lower operating voltages, which are typically in the order of $1 \mathrm{~V}$.

As the energy of a capacitor is related to the capacitance and voltage of the device by

$$
\mathrm{E}=\frac{\mathrm{CV}^{2}}{2}
$$

where E is the energy stored $(\mathrm{J}), \mathrm{C}$ is the capacitance $(\mathrm{F})$ and $\mathrm{V}$ is the cell voltage $(\mathrm{V})$, many efforts have been made to compensate for the limited width of the potential window in aqueous-based electrochemical capacitors. Some previously known electroactive materials have recently been examined due to their high capacitance. These materials have included transition metal oxides, ${ }^{5-14}$ with amorphous hydrated $\mathrm{RuO}_{2}$ exhibiting the highest reported capacitance $\left(\sim 760 \mathrm{~F} / \mathrm{g}\right.$ in acidic electrolytes $\left.{ }^{5,15-17}\right)$, and can contribute to the positive limit of the potential window, with some studies reporting an extension of the potential window to $1.4 \mathrm{~V},{ }^{18-20}$ thereby achieving higher energy and power densities when compared to other systems. However, despite these improvements, the cell voltage is still too low to achieve satisfactory energy for practical applications. Additionally, the prohibitive cost of ruthenium, especially as its oxide, low pore volume, and toxicity of such compounds contributes to the impracticability of capacitors made from such materials. Oxides of other transition metals, such as manganese, have been widely investigated as the positive electrode in asymmetric systems, with a carbon material (such as activated carbon or graphite) as the negative electrode. ${ }^{21}$

Manganese dioxide (commonly referred as $\mathrm{MnO}_{2}$, but more accurately a non-stoichiometric arrangement of $\left[\mathrm{Mn}\left(\mathrm{O}^{2-}, \mathrm{OH}^{-}\right.\right.$, $\left.\left.\mathrm{H}_{2} \mathrm{O}\right)_{6}\right]^{\mathrm{z}+}$ octahedra) have a long history as cathode materials in aqueous and non-aqueous batteries, and have proven to be a promising category of electrochemical capacitor electrode materials. Previous findings have shown that films of manganese dioxide deposited using chronoamperometry exhibited specific capacitances $>2000$ $\mathrm{F} / \mathrm{g}{ }^{22}$ In these cases it was deduced that careful control of the mass transport characteristics by varying certain parameters during deposition was important - specifically, it was demonstrated that lower deposition voltages, and high acid/low $\mathrm{Mn}$ (II) concentrations in the electrolyte produced the best performing electrodes. These conditions led to slower mass transport, which apparently has a favourable effect on material morphology. 
This work.-This study aims to compare the performance of electrodes deposited using chronoamperometry (constant potential), to those using a galvanostatic (constant current) deposition protocol. The aim is to pass equivalent amounts of charge during deposition, but vary the current density. In addition to material performance, insight into the manganese dioxide deposition mechanism will also be achieved.

\section{Experimental}

Electrodeposition.-All deposition solutions contained a combination of $0.01 \mathrm{M} \mathrm{MnSO}_{4}$ (AnalaR, >98\%) and $0.1 \mathrm{M} \mathrm{H}_{2} \mathrm{SO}_{4}$ (Ajax Finechem, 95-98\%). The working electrodes used during deposition and cycling were Radiometer Analytical XM150 platinum electrodes $\left(1 \mathrm{~cm}\right.$ diameter, $0.785 \mathrm{~cm}^{2}$ area), with a saturated calomel electrode (SCE) as a reference, and a graphite rod as a counter electrode. Initially, so as to determine appropriate voltages for deposition, a linear sweep voltammetry experiment was performed using a Perkin-Elmer VMP multichannel potentiostat/galvanostat, and the cell voltage was swept from the open circuit potential $(\sim 0.6 \mathrm{~V})$ to $0 \mathrm{~V}$, and then to $1.6 \mathrm{~V}$ (vs. SCE) at a scan rate of $5 \mathrm{mV} / \mathrm{s}$.

After obtaining the linear sweep voltammogram, a potential in the non-diffusion limited region was selected $(1.2 \mathrm{~V})$, and was used as the step potential (from the open circuit potential) in a chronoamperometic deposition. Chronoamperometric deposition was performed initially for $30 \mathrm{~s}$, and the total charge passed during this deposition was calculated via integration of the current vs. time plot. To compare the effects of varying the electrodeposition method, galvanostatic depositions were also performed. Here the equivalent amount of charge passed during the $30 \mathrm{~s}$ CA deposition was passed over varying times ranging from 1-60 s. This process was repeated for charges equivalent to 5,15 and $60 \mathrm{~s}$ of chronoamperometry at $1.2 \mathrm{~V}$.

Electrochemical cycling.-The electrodeposited $\mathrm{MnO}_{2}$ was then electrochemically tested via cyclic voltammetry. After deposition, whether by chronoamperometry or galvanostatic means, the deposited films were washed thoroughly with Milli-Q water, patted dry with paper towel and then transferred to the cycling electrolyte $(0.5$ $\mathrm{M} \mathrm{Na}_{2} \mathrm{SO}_{4}$ ) together with a saturated calomel reference and graphite counter electrode. The cell was cycled between 0-0.8 V vs SCE for 50 cycles at a scan rate of $5 \mathrm{mV} / \mathrm{s}$, using the same Perkin-Elmer VMP as for the deposition.

\section{Results and Discussion}

Manganese dioxide electrodeposition mechanism.-Since the nature of the manganese dioxide materials we will be producing will be dependent on the conditions under which they were made, it is appropriate to begin by presenting the currently accepted mechanisms for manganese dioxide electrodeposition. There are several deposition mechanisms that have been proposed for the oxidation of $\mathrm{Mn}^{2+}$ to $\mathrm{MnO}_{2}$. The two main pathways are dependent on acid concentration, and are hence referred to as the low acid and high acid mechanisms. As shown as Path A in Fig. 1, the low acid mechanism involves oxidation of $\mathrm{Mn}^{2+}$ to $\mathrm{Mn}^{3+}$, hydrolysis of the $\mathrm{Mn}^{3+}$ to precipitate $\mathrm{MnOOH}$ on the electrode surface, and then further solid state oxidation to give $\mathrm{MnO}_{2}$. The high acid process (Path C in Fig. 1) also begins with an oxidation step; however, the next step is a disproportionation $\left(2 \mathrm{Mn}^{3+} \rightarrow \mathrm{Mn}^{4+}+\mathrm{Mn}^{2+}\right)$, and a final hydrolysis of the resulting $\mathrm{Mn}^{4+}$ to give $\mathrm{MnO}_{2}$. While possible, there has been no experimental evidence presented in the literature to support the direct two electron oxidation of $\mathrm{Mn}^{2+}$ to $\mathrm{Mn}^{4+}$ (Path B in Fig. 1).

Electrodeposition data.-As outlined in the Experimental section, the appropriate non-diffusion limited voltage had to be ascertained from a linear sweep voltammogram in the chosen electrolyte. The linear-sweep voltammogram obtained from $0.1 \mathrm{M}$ $\mathrm{H}_{2} \mathrm{SO}_{4}+0.01 \mathrm{M} \mathrm{MnSO}_{4}$ is shown in Fig. 2. This electrolyte was chosen because of its ability to produce high performing

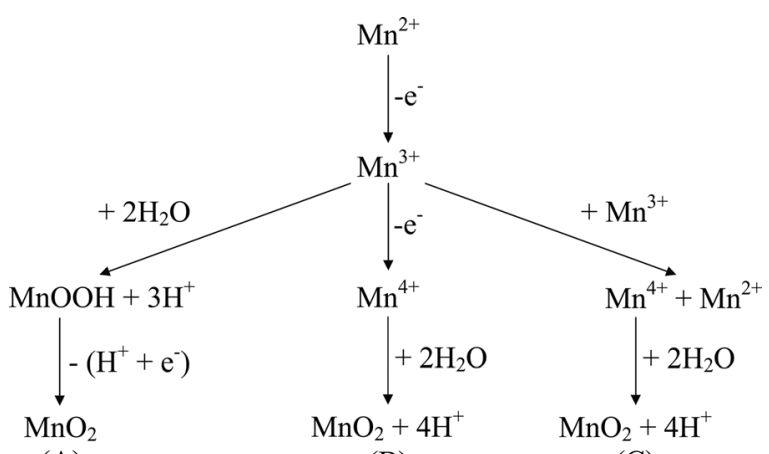

(A)

(B)

(C)

Figure 1. Mechanistic pathways for the electrodeposition of manganese dioxide.

electrodeposited manganese dioxide, as per our previous work in this area. $^{22}$ The voltammogram in Fig. 2 shows two closely overlapping anodic waves at $\sim 1.253$ and $\sim 1.364 \mathrm{~V}$ which correspond to the two stage oxidation of $\mathrm{Mn}^{2+}$ to $\mathrm{MnO}_{2}$. What is interesting, and very relevant for our ability to estimate the amount of electrodeposited manganese dioxide, is that at slightly higher and overlapping potentials there is evidence for the oxygen evolution reaction occurring. However, since we have chosen a non-diffusion limited potential $(1.2 \mathrm{~V}$ vs SCE) to carry out our chronoamperometry experiments, the contributions made by oxygen evolution to the total anodic charge passed are expected to be minimal.

A typical chronoamperogram (ivs $t$ ) is shown in Fig. 3, in which case the potential was stepped from the open circuit potential to $1.2 \mathrm{~V}$ vs SCE for $30 \mathrm{~s}$. It shows the expected spike in current after the imposition of the step potential, as well as the subsequent decay in current with time. What was unexpected was the slight increase in current after longer deposition times. This phenomenon, after consideration of the details of a chronoamperometry experiment, and the factors that influence its behaviour, ${ }^{23}$ is believed to be due to a changing electrode area as a result of manganese dioxide deposition. Similar results have been obtained in our research group with the electrodeposition of poly(terthiophene). ${ }^{24}$ For the data shown in Fig. 3 it is not possible to estimate the increase in area of the electrode (as was done in the literature case) because the conditions

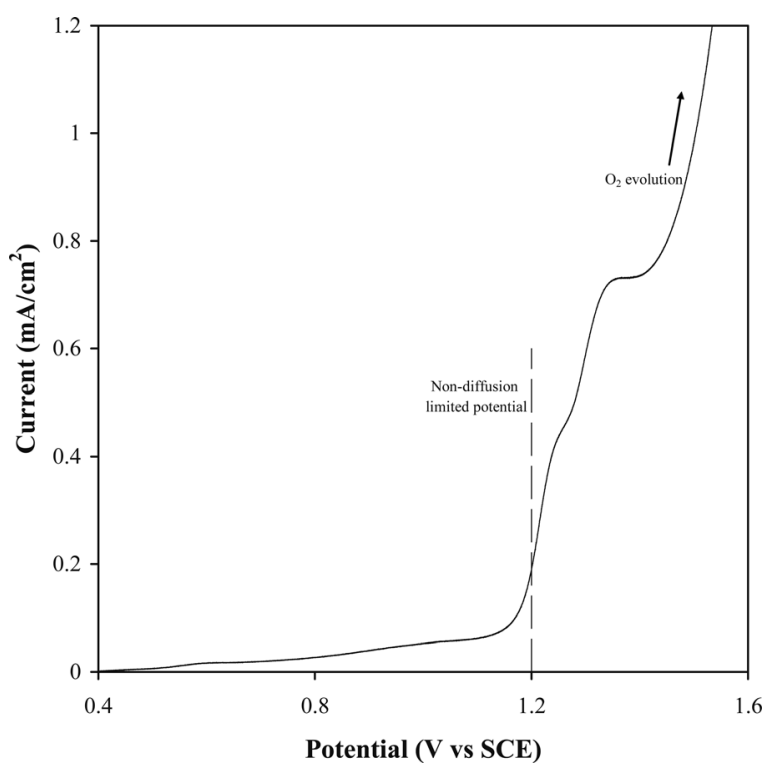

Figure 2. Linear sweep voltammogram for the electrodeposition of manganese dioxide from an electrolyte of $0.01 \mathrm{M} \mathrm{MnSO}_{4}+0.1 \mathrm{M} \mathrm{H}_{2} \mathrm{SO}_{4}$. Scan rate $5 \mathrm{mV} / \mathrm{s}$. 


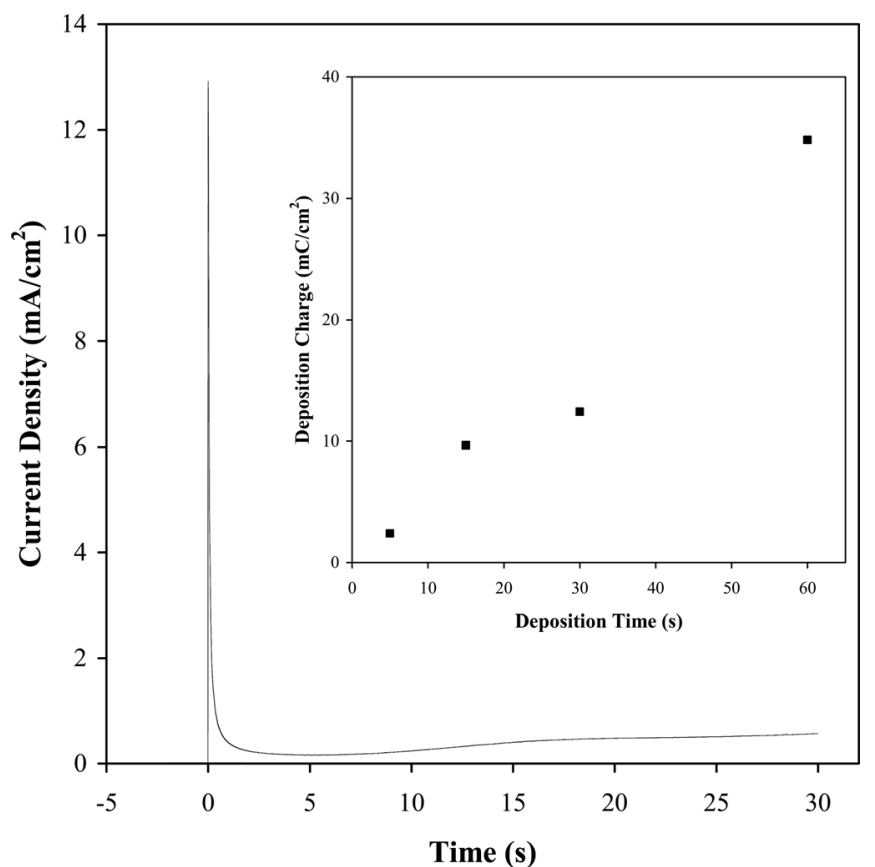

Figure 3. Chronoamperometric data (i vs $\mathrm{t}$ ) from an electrolyte of $0.01 \mathrm{M}$ $\mathrm{MnSO}_{4}+0.1 \mathrm{M} \mathrm{H}_{2} \mathrm{SO}_{4}$. Step potential $1.2 \mathrm{~V}$ vs SCE. Inset: Charge passed during each deposition.

under which electrodeposition occurred were not diffusion limited, and hence the concentration of $\mathrm{Mn}^{2+}$ at the electrode surface was not zero, and variable. As such, the Cottrell equation for chronoamperometry experiments ${ }^{23}$ could not be applied.

After chronoamperometry was performed at 5, 15, 30 and $60 \mathrm{~s}$, the charge passed during each deposition was calculated via numerical integration of the current passed during deposition. This data is included as an inset in Fig. 3, and also in Table I. This table also contains the calculated theoretical mass of manganese dioxide deposited based on the charge passed. To demonstrate the conversion from chronoamperometry to galvanostatic deposition, the charge passed during the $30 \mathrm{~s}$ deposition experiment was $12.425 \mathrm{mC} / \mathrm{cm}^{2}$, so for galvanostatic deposition with the same charge, and for example a deposition time of $30 \mathrm{~s}$, the constant current will be equal to $0.414 \mathrm{~mA} / \mathrm{cm}^{2}$. Table I also contains a listing of the galvanostatic currents and times to match the charge passed during each of the chronoamperometry experiments.

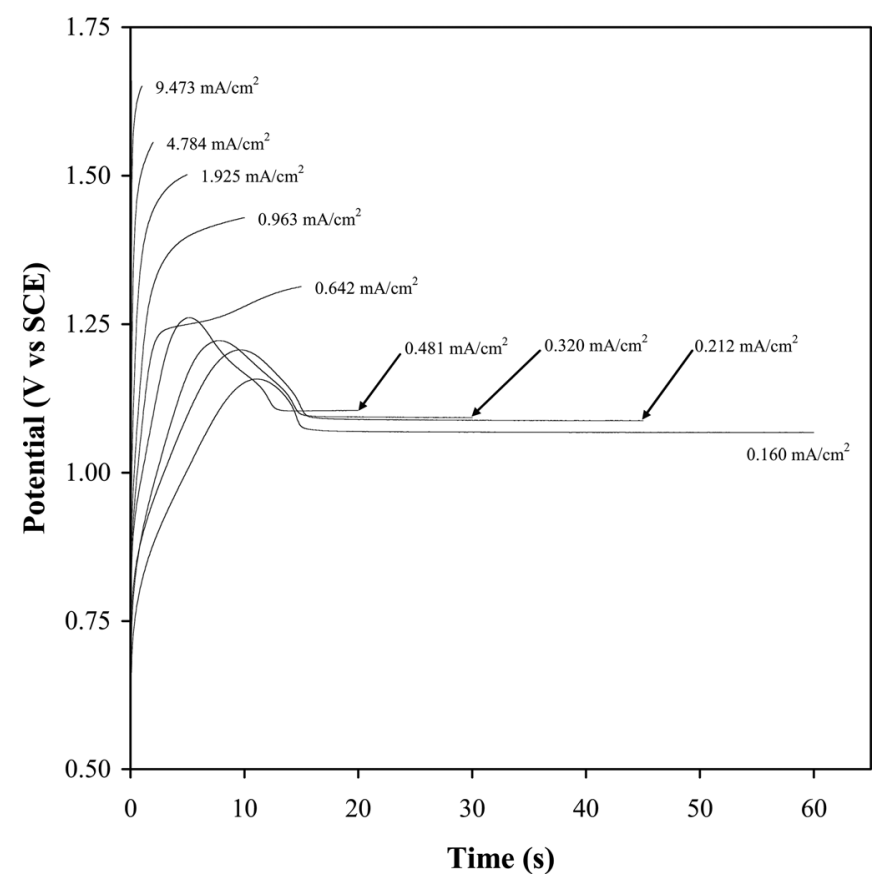

Figure 4. Potential (vs. SCE) versus deposition time for the galvanostatic depositions equivalent to $15 \mathrm{~s}$ chronoamperometry in $0.1 \mathrm{M} \mathrm{H}_{2} \mathrm{SO}_{4}+0.01 \mathrm{M}$ $\mathrm{MnSO}_{4}$. Current densities used are indicated on each data set.

As a result of holding the current density at a constant value, as opposed to a constant potential during chronoamperometry, the potential during galvanostatic deposition changes over time. Examples of this for galvanostatic depositions equivalent to $15 \mathrm{~s}$ of chronoamperometry in $0.1 \mathrm{M} \mathrm{H}_{2} \mathrm{SO}_{4}+0.01 \mathrm{M} \mathrm{MnSO}_{4}$ are shown in Fig. 4, where an interesting trend in the potential data can be seen. For short timeframe deposition experiments $(<15 \mathrm{~s})$, and hence higher current density experiments, the electrode potential increases continually, finishing within the range $1.25-1.65 \mathrm{~V}$ depending on the current density used. For longer duration experiments $(>15 \mathrm{~s})$, and hence lower current densities, the potential exhibits a maximum, after which it drops and eventually plateaus. Once the plateau was reached the electrode remains at this potential for the remainder of the deposition.

It is appropriate at this time to consider what the potential changes represent in terms of the electrodeposition mechanisms shown in Fig. 1. With the electrolyte conditions we are using, in

Table I. Data pertaining to the chronoamperometric and galvanostatic depositions of manganese dioxide.

Chronoamperometry

\begin{tabular}{|c|c|c|c|c|}
\hline Time (s) & 5 & 15 & 30 & 60 \\
\hline Charge $(\mathrm{mC})$ & 1.88 & 7.59 & 9.75 & 27.34 \\
\hline Mass $\mathrm{MnO}_{2}(\mu \mathrm{g})$ & 0.848 & 3.419 & 4.394 & 12.315 \\
\hline \multicolumn{5}{|c|}{ Galvanostatic deposition } \\
\hline Time (s) & \multicolumn{4}{|c|}{ Current $\left(\mathrm{mA} / \mathrm{cm}^{2}\right)$} \\
\hline 1 & 2.348 & 9.473 & 12.176 & \\
\hline 2 & 1.186 & 4.784 & 6.150 & $12.646(2.74 \mathrm{~s})$ \\
\hline 5 & 0.476 & 1.925 & 2.474 & 6.698 \\
\hline 10 & 0.237 & 0.963 & 1.239 & 3.355 \\
\hline 15 & 0.158 & 0.642 & 0.781 & 2.238 \\
\hline 20 & 0.119 & 0.481 & 0.495 & 1.343 \\
\hline 25 & 0.094 & & 0.619 & 1.119 \\
\hline 30 & 0.079 & 0.320 & 0.412 & 0.746 \\
\hline 45 & 0.051 & 0.212 & 0.414 & 0.580 \\
\hline 60 & 0.039 & 0.160 & 0.274 & 0.560 \\
\hline Ave. $\mathrm{MnO}_{2}$ Mass $(\mu \mathrm{g})$ & $0.833 \pm 0.009$ & $3.391 \pm 0.018$ & $4.343 \pm 0.071$ & $11.962 \pm 0.184$ \\
\hline
\end{tabular}


particular the high acid concentration $\left(0.1 \mathrm{M} \mathrm{H}_{2} \mathrm{SO}_{4}\right)$, it is reasonable to presume that any $\mathrm{Mn}^{3+}$ intermediate will have a relatively long lifetime in solution before either hydrolysis or disproportionation occurs. What we propose is that at the start of the deposition experiment the rapid increase in potential is due primarily to the formation of a soluble $\mathrm{Mn}^{3+}$ intermediate. The maximum in the electrode potential is obtained as a result of the $\mathrm{Mn}^{3+}$ becoming supersaturated in the vicinity of the electrode, after which either hydrolysis (to $\mathrm{MnOOH}$, with subsequent solid state oxidation to $\mathrm{MnO}_{2}$ ) or disproportionation (to form $\mathrm{Mn}^{2+}$ in solution and solid $\mathrm{MnO}_{2}$ on the electrode) leads to the deposition of a lower potential solid material on the electrode surface. The plateau represents the quasi-equilibrium two phase potential between the solid manganese dioxide produced and the bulk electrolyte concentration.

With this description of the mechanism occurring at the electrode surface we are now in the position of being able to interpret the implications of a changing potential on the properties of the deposited manganese dioxide, in particular the amount of material deposited. In the first case, given the acid concentration of the electrolyte being used it is possible that the soluble $\mathrm{Mn}^{3+}$ intermediate formed may not have sufficient time to either hydrolyse or disproportionate and deposit onto the electrode before the deposition experiment has been completed. In fact, the increasing potential seen in Fig. 4 would indicate that little deposition of a solid material onto the electrode has occurred. The impact of this is that charge is effectively lost to the electrolyte without leading to a manganese dioxide deposit on the electrode. Furthermore, it means that we will overestimate the amount of manganese dioxide on the electrode surface because of this loss of charge (due to uncaptured $\mathrm{Mn}^{3+}$ ) to the bulk of the electrolyte.

Another complicating factor associated with constant current deposition and hence an uncontrolled potential is the fact that competing reactions can also occur. From the linear sweep voltammogram in Fig. 2 it was highlighted that manganese dioxide deposition overlaps with oxygen evolution on the anode. Again, for the short time frame, high current depositions oxygen evolution will be competing with manganese dioxide electrodeposition. This overlapping reaction can consume charge that would otherwise be associated with manganese dioxide deposition, again leading to an overestimate of the amount of material deposited.

In an attempt to confirm the mass of manganese dioxide deposited onto the substrate duplicate electrodes were prepared and washed thoroughly with Milli-Q ultra-pure water to remove any remaining plating electrolyte. The electrode was then redissolved in acidified (5\% v/ $\left.\mathrm{v} \mathrm{H}_{2} \mathrm{SO}_{4}\right) \mathrm{H}_{2} \mathrm{O}_{2}$, with the resultant solution analysed for total manganese content using ICP-AES (Varian Liberty Series II). Over the entire range of manganese dioxide electrode materials prepared it was determined that using the i-t integral (charge) to determine the amount of $\mathrm{MnO}_{2}$ underestimated the electrode mass by $5-10 \%$. Nevertheless, for the purposes of this study, the mass calculation using charge was still employed.

As a final comment in this section, it is anticipated that the current used for deposition will dramatically affect the morphology of the electrodeposited manganese dioxide, and hence the performance of the material as a supercapacitor electrode. From our past experience with electrodeposited manganese dioxide ${ }^{22}$ we expect that the extent of disorder within the deposit will be greatest for the higher current density depositions, while the use of a low current density will lead to a relatively dense, low porosity deposit. These statements on morphology are supported by experimental evidence such as cation vacancy fraction, BET surface area, and crystal structure on bulk deposits of manganese dioxide. For thin films of manganese dioxide, such as we are producing here, characterizing their properties is exceedingly difficult since there is very little material available for characterization. In fact, the electrochemical cycling as a supercapacitor electrode in a suitable electrolyte is probably the most appropriate method for evaluating the deposited material morphology.

Cycling performance.-Given that during the course of this study the conditions used during deposition were similar; i.e., all deposition experiments were performed at the same temperature $\left(22 \pm 1^{\circ} \mathrm{C}\right)$ and using the same electrolyte conditions, it is reasonable to assume that the crystallographic phase of manganese dioxide formed during deposition is the same. Therefore, any differences in cycling performance are due to differences in the morphology of the electrode film. It is also reasonable to expect that an electrode with a relatively high surface area and porosity will result in a higher capacitance than a denser electrode with a reduced surface area. As a result of this, any phenomena that is seen to occur in cycling data is related to the morphology, which in turn is due to the differing conditions during deposition, most notably the current and voltages used or achieved during deposition.

Figure 5 shows typical cyclic voltammograms of an electrodeposited manganese dioxide prepared in this work. As can be seen in the figure, the voltammogram exhibits the expected "box" shape of a supercapacitive electrode material. Furthermore, there is a high degree of consistency with cycling as shown by the 25th cycle in Fig. 5, as well as the anodic:cathodic charge ratio (inset), which is essentially constant over the cycle range examined. To evaluate performance we have determined the specific capacitance for each electrode after 50 cycles. The mass of manganese dioxide used for the normalization procedure was determined from the amount of charge passed during the electrodeposition experiment, assuming that $\mathrm{MnO}_{2}$ was the stoichiometric product. As was mentioned previously, there may be complications associated with this estimate of mass, particularly for the short time frame, high current density experiments, where some of the charge may be lost due the solubility and stability of $\mathrm{Mn}^{3+}$ intermediate species, as well as from the competing oxygen evolution reaction. Calculating the specific capacitance enables a comparison firstly between the use of different deposition current densities, as well as whether the deposition was carried out chronoamperometrically or galvanostatically.

Figure 6 shows the relationship between deposition time and specific capacitance for all of the experiments conducted. To begin, the chronoamperometric data in this figure (large squares) shows a very clear exponential drop in specific capacitance as the deposition time, and hence deposition charge, was increased. This is consistent with our previous work in the area, and is an indication of how the density of the deposit increases as the deposition time increases.

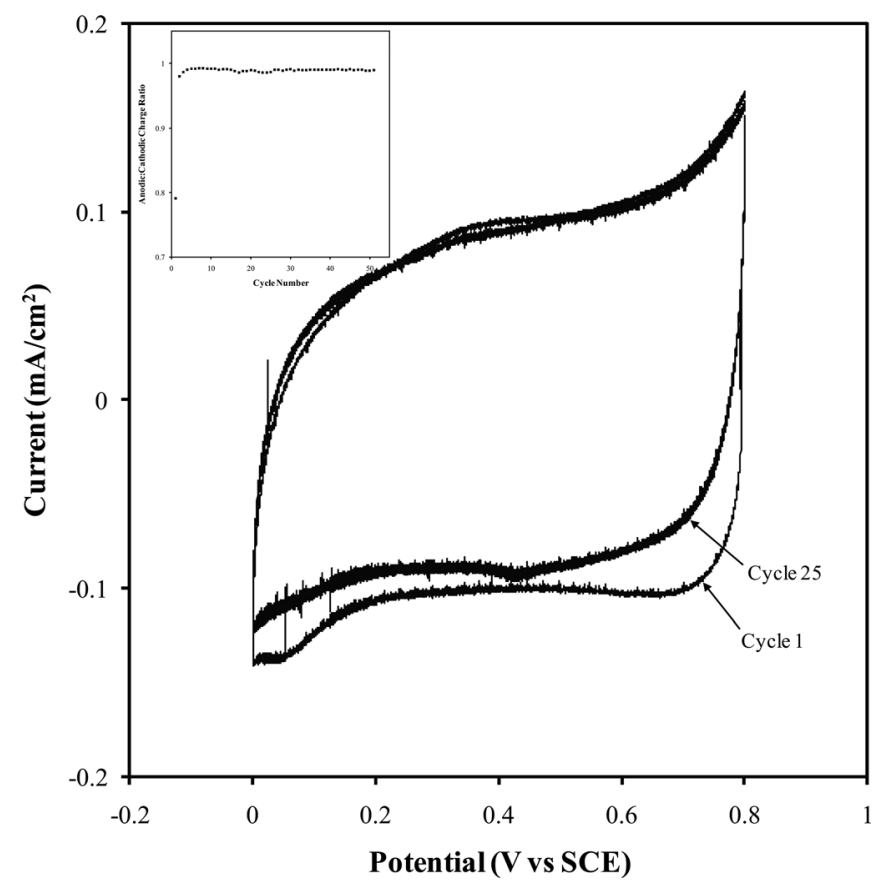

Figure 5. Typical cyclic voltammogram from an electrode prepared in this work. Inset: Anodic:cathodic charge ratio for the corresponding electrode. 


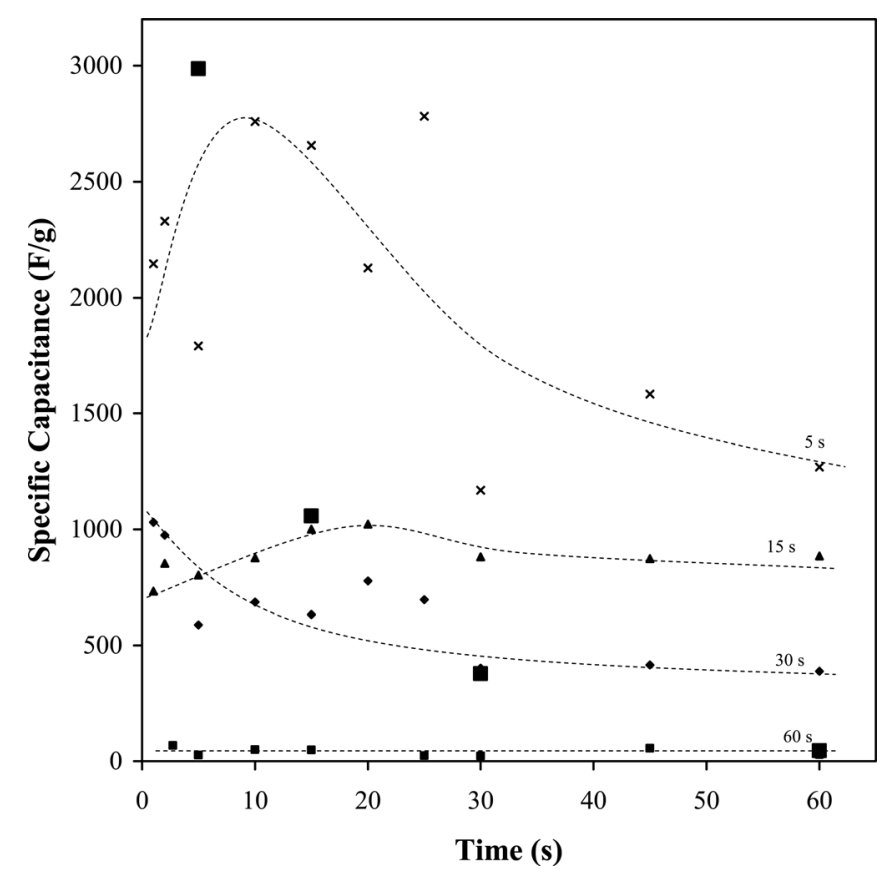

Figure 6. Specific capacitance for each electrode. (ם) Chronoamperometric deposition, and galvanostatic data corresponding to (ם) $60 \mathrm{~s},(\diamond) 30 \mathrm{~s},(\boldsymbol{\Delta})$ $15 \mathrm{~s}$, and $(\times) 5 \mathrm{~s}$ of chronoamperometric charge.

The chronoamperometry experiment conducted over $60 \mathrm{~s}(34.82$ $\mathrm{mC} / \mathrm{cm}^{2}$ ) led to a very low specific capacitance, with only $45 \mathrm{~F} / \mathrm{g}$ being achieved. With such a relatively long time frame experiment, with a relatively low current density used, it was to be expected that this deposit was quite dense and hence of low capacitance. For all of the corresponding galvanostatic depositions, the specific capacitance was similar, and relatively constant irrespective of the deposition current density used. For the short time frame deposition experiments done here, we may have overestimated the amount of manganese dioxide present, and so the specific capacitance here may indeed be more enhanced than what was recorded. Nevertheless, the average specific capacitance under these circumstances was $42 \pm 5 \mathrm{~F} / \mathrm{g}$ with no trends apparent in the data.

For the $30 \mathrm{~s}$ chronoamperometry experiment $\left(12.42 \mathrm{mC} / \mathrm{cm}^{2}\right.$ of charge passed during deposition) a specific capacitance of $378 \mathrm{~F} / \mathrm{g}$ was achieved, with the equivalent galvanostatic deposit $(0.412 \mathrm{~mA} /$ $\mathrm{cm}^{2}$ for $\left.30 \mathrm{~s}\right)$ resulting in a similar specific capacitance $(402 \mathrm{~F} / \mathrm{g})$. These values are very similar to those obtained from the use of lower deposition current densities (longer deposition times); i.e., $417 \mathrm{~F} / \mathrm{g}$ for the $45 \mathrm{~s}$ deposition $\left(0.274 \mathrm{~mA} / \mathrm{cm}^{2}\right)$ and $388 \mathrm{~F} / \mathrm{g}$ for the $60 \mathrm{~s}$ deposition $\left(0.205 \mathrm{~mA} / \mathrm{cm}^{2}\right)$. It is to be expected, based on our previous work, ${ }^{22}$ that lower current densities result in a denser electrode film, and hence a lower specific capacitance. When the deposition time was much shorter, considerable improvements in capacitive performance were observed. Indeed, a maximum specific capacitance was observed at for a $1 \mathrm{~s}$ deposition $\left(12.176 \mathrm{~mA} / \mathrm{cm}^{2}\right)$, with a value of $1032 \mathrm{~F} / \mathrm{g}$, which decreased steadily as the deposition time increased until the aforementioned values for longer deposition times were reached. What this data is beginning to suggest is that for very short timeframe deposition experiments only the nuclei of manganese dioxide crystallites are being produced on the substrate surface. These nuclei have a very high surface area and hence when cycled, lead to a very high specific capacitance. With continued deposition, crystal growth predominates effectively closing off pores, decreasing surface area, leading to a denser deposit and hence a much lower specific capacitance.

The deposit made using a $15 \mathrm{~s}$ chronoamperometry experiment $\left(9.67 \mathrm{mC} / \mathrm{cm}^{2}\right)$ had a specific capacitance of $1059 \mathrm{~F} / \mathrm{g}$. For the corresponding galvanostatically prepared deposits the trend in specific capacitance with deposition time was quite interesting. For longer time frame deposits ( $>20 \mathrm{~s}$ ) the specific capacitance decayed exponentially from $1023 \mathrm{~F} / \mathrm{g}$ (for a $20 \mathrm{~s}$ deposit using $0.481 \mathrm{~mA} / \mathrm{cm}^{2}$ ) as the deposition time increased. However, for shorter duration experiments the specific capacitance was seen to increase from $733 \mathrm{~F} / \mathrm{g}$ (for a $1 \mathrm{~s}$ deposit using $9.47 \mathrm{~mA} / \mathrm{cm}^{2}$ ) up to a maximum at $20 \mathrm{~s}$. We believe that this phenomenon is a result of an inaccurate estimate of the amount of manganese dioxide deposited onto the electrode substrate. Under these circumstances we have a relatively small amount of charge passed during deposition, and if some of that is lost as a result of soluble $\mathrm{Mn}^{3+}$ formation, or from the competing oxygen evolution reaction, then the overestimate of manganese dioxide deposited onto the substrate will be much more significant. Given that for these shorter timeframe experiments the deposition potential is also high, indicating either excessive $\mathrm{Mn}^{3+}$ formation or oxygen evolution (see Fig. 4) as a likely explanation.

The same result is also apparent for the $5 \mathrm{~s}$ chronoamperometry experiment $\left(2.40 \mathrm{mC} / \mathrm{cm}^{2}\right.$ of deposition charge) which generated a specific capacitance of $2986 \mathrm{~F} / \mathrm{g}$. All of the galvanostatic depositions carried out with the same charge were inferior to the chronoamperometrically prepared sample; however, a maximum in specific capacitance was also observed here. As before, this maximum is due to the combination of a small amount of deposition charge and the high current density used for the short time frame experiments leading to an overestimate of the amount of manganese dioxide deposited.

\section{Summary and Conclusions}

In this study we have compared the ability of chronoamperometry and galvanostatic methods to electrodeposit thin films of manganese dioxide for use as supercapacitor electrodes. The following points are significant outcomes from the work:

(i) During chronoamperometric deposition an increase in the current towards the end of the experiment is evidence of the electrode area increasing as a result of the manganese dioxide morphology.

(ii) The mechanism of manganese dioxide electrodeposition plays a significant role in determining the properties of the resultant deposit. From the data collected, chronoamperometry is the preferred method for smaller amounts of charge (i.e. 1.88 and 7.59 $\mathrm{mC})$, where as for larger amounts of charge (9.75 and $27.34 \mathrm{mC})$, the performance of galvanostatically prepared electrodes is comparable to those prepared from chronoamperometry.

(iii) For these conditions (short timeframe galvanostatic deposition with high current density and low charge deposits) complications were also identified relating to the mass of electrodeposited manganese dioxide on the substrate. Under these circumstances, a significant proportion of the $\mathrm{Mn}^{3+}$ intermediate was lost to the electrolyte leading to an overestimate of the amount of deposited manganese dioxide. Oxygen evolution as a competing anodic reaction can also contribute to an inaccurate estimate of deposited manganese dioxide.

(iv) The specific capacitance obtained for each electrode was used as a tool to characterize the morphology of the deposited manganese dioxide. Again, short timeframe experiments were preferred because of their ability to produce high performance electrodes, implying a deposit with high surface area and porosity. Longer time frame experiments led to poor performing electrodes, indicating much denser, less porous films.

\section{Acknowledgments}

AC acknowledges the financial support provided by the University of Newcastle and CSIRO Energy Technology in the form of a postgraduate scholarship.

\section{References}

1. http://www.eia.doe.gov/oiaf/ieo/world.html, accessed April 14, 2011.

2. A. Sari and K. Kaygusuz, Sol. Energy, 71, 365 (2001). 
3. R. F. Post, K. Fowler, and S. F. Post, Proc. IEEE, 81, 462 (1993).

4. D. Ragone in Proceedings of Society of Automotive Engineers Conference, Detroit, MI, May (1968).

5. J. P. Zheng and T. R. Jow, J. Electrochem. Soc., 142, L6 (1995).

6. H. H. Kim and K. B. Kim, Electrochem. Solid-State Lett., 4, A62 (2001).

7. J. K. Chang and W. T. Tsai, J. Electrochem. Soc., 150, A1333 (2003).

8. C. C. Hu and T. W. Tsou, J. Power Sources, 115, 179 (2003).

9. J. W. Long, A. L. Young, and D. R. Rolison, J Electrochem. Soc., 150, A1161 (2003).

10. H. Kim and B. N. Popov, J. Electrochem. Soc., 150, D56 (2003).

11. R. N. Reddy and R. G. Reddy, J. Power Sources, 124, 330 (2003).

12. N. L. Wu, Mater. Chem. Phys., 75, 6 (2002).

13. V. Srinivasan and J. W. Weidner, J. Electrochem. Soc., 144, L220 (1997).

14. H. K. Kim, T. Y. Seong, J. H. Lim, W. I. Cho, and Y. S. Yoon, J. Power Sources, 102, 167 (2001).
15. J. P. Zheng, P. J. Cygan, and T. R. Jow, J. Electrochem. Soc., 142, 2699 (1995).

16. J. P. Zheng, Electrochem. Solid-State Lett., 2, 359 (1999).

17. M. C. Santos, A. J. Terezo, V. C. Fernandes, E. C. Pereira, and L. O. S. Bullhoes, J. Solid State Electrochem., 9, 91 (2005).

18. Y.-G. Wang, Z.-D. Wang, and Y.-Y. Xia, Electrochim. Acta, 50, 5641 (2005).

19. B.-O. Park, C. D. Lokhande, H.-S. Park, K.-D. Jung, and O.-S. Joo, J. Mater. Sci., 39, 4313 (2004)

20. X.-F. Wang, Z. You, and D.-B. Ruan, Chin. J. Chem., 24, 1126 (2006).

21. M. S. Hong, S. H. Lee, and S. W. Kim, Electrochem. Solid-State Lett., 5, A227 (2002).

22. A. D. Cross, A. Morel, A. Cormie, A. F. Hollenkamp, and S. W. Donne, J. Power Sources, 196, 7847 (2011).

23. A. J. Bard and L. R. Faulkner, Fundamentals of Electrochemistry, 2nd ed., John Wiley \& Sons: New York, (2001)

24. D. Wakeham, S. W. Donne, W. J. Belcher, and P. C. Dastoor, Synth. Met., 158, 661 (2008). 